\section{Rapid Hospital Room Decontamination Using Ultraviolet (UV) Light with a Nanostructured UV-Reflective Wall Coating}

William A. Rutala, PhD, MPH; ${ }^{1,2}$

Maria F. Gergen, MT(ASCP); ${ }^{1}$ Brian M. Tande, PhD; ${ }^{3}$

David J. Weber, MD, MPH ${ }^{1,2}$

\begin{abstract}
We tested the ability of an ultraviolet C (UV-C)-reflective wall coating to reduce the time necessary to decontaminate a room using a UV-C-emitting device (Tru-D SmartUVC). The reflective wall coating provided the following time reductions for decontamination: for methicillin-resistant Staphylococcus aureus, from 25 minutes 13 seconds to 5 minutes 3 seconds $(P<.05)$, and for Clostridium difficile spores, from 43 minutes 42 seconds to 9 minutes 24 seconds $(P<.05)$.
\end{abstract}

Infect Control Hosp Epidemiol 2013;34(5):527-529

Surface disinfection of noncritical surfaces and equipment is normally performed by manually applying a liquid disinfectant to surfaces or objects with a cloth, disposable wipe, or mop. Effective environmental disinfection in healthcare facilities is essential for reducing environment-mediated infection transmission via contaminated hands of healthcare personnel or environmental surfaces. Recent studies have identified substantial opportunities in hospitals to improve the cleaning and disinfection of hospital room surfaces. ${ }^{1}$ For example, of 20,646 standardized environmental surfaces (14 types of objects), only $9,910(48 \%)$ were cleaned at terminal room cleaning according to institutional cleaning policies. ${ }^{1}$ Epidemiologic studies have shown that patients admitted to rooms previously occupied by individuals infected or colonized with methicillin-resistant Staphylococcus aureus (MRSA), ${ }^{2}$ vancomycin-resistant Enterococcus (VRE), ${ }^{2,3}$ or Clostridium difficile ${ }^{4}$ are at significant risk of acquiring these organisms. These data have led to efforts to improve surface disinfection practices and the development of room decontamination units that avoid the problems associated with manual disinfection. ${ }^{5}$

We investigated a room decontamination unit that uses ultraviolet C (UV-C) energy $(254 \mathrm{~nm})$. The unit is fully automated and is activated by a handheld remote, and the room ventilation does not need to be modified. It measures UV-C reflected from walls, ceilings, floors, and items in the room and calculates the time required to deliver the programmed lethal dose for pathogens. ${ }^{6}$ Following decontamination, it will power down, and an audible alarm will notify the operator. The goal of this study was to assess the time required for a UV-C room decontamination unit to kill important health- care-associated pathogens (ie, MRSA and C. difficile) in a room with standard white paint-coated walls versus walls coated with an agent specifically formulated to be reflective of UV-C wavelengths.

\section{METHODS}

A single UV-C device was investigated (Tru-D SmartUVC; Lumalier); it can be set to deliver a minimum reflected dose of $22,000 \mu \mathrm{Ws} / \mathrm{cm}^{2}$ for $C$. difficile spores and $12,000 \mu \mathrm{Ws} /$ $\mathrm{cm}^{2}$ for vegetative bacteria. All testing (with the exception of 3 cycles in a nonreflective room) was done in a single patient hospital room (117- $\mathrm{ft}^{2}$ room plus $13 \mathrm{ft}^{2}$ for the bathroom). The cycle time to achieve microbial killing was determined in this room before and after the room was coated with an agent designed to maximize the UV-C reflectivity. The coating (Lumacept) is formulated using nanoscale inorganic oxides whose crystal structures are transparent to UV-C. It also contains polymer binders and functional additives with chemical structures that are minimally absorbent of UV-C. Standard paint is $3 \%-7 \%$ UV reflective, while the special coating is $65 \%$ UV reflective at $254 \mathrm{~nm}$. The coating is white in appearance and can be applied with a brush or roller in the same way as any common interior latex paint. The cost to coat the walls of the room and bathroom used in this study (approximately $12.1 \mathrm{~m}^{2}$ ) was estimated to be less than $\$ 300$.

Testing was performed using Formica sheets (approximately 3 in. $\times 3$ in.), with a template of a Rodac plate (approximately $25 \mathrm{~cm}^{2}$; Becton Dickinson) drawn on each sheet. MRSA was grown on sheep blood agar. The $C$. difficile spore preparation was stored in Dulbecco's modified Eagle medium (HyClone), and serial dilutions were made with trypticase soy broth (Remel). A $10-\mu \mathrm{L}$ inoculum containing approximately $10^{4}-10^{5}$ organisms/Rodac template of the 2 test organisms was spread separately on the Formica sheet by use of a sterile glass hockey loop. The test organisms were $C$. difficile spores (BI strain) and a clinical isolate of MRSA (USA300 strain). After our templates were inoculated, the Formica sheets were left to dry for a minimum of 10 minutes at room temperature and were then placed in 10 locations throughout the patient room (ie, the far side of the bedside table, facing the wall; the top of the bed; the closet door; the top of the toilet seat; the back of the chair; the floor [right side of the bed]; the foot of the bed, facing the door; the side of the sink, facing the bedside table; the back of the computer, facing the wall; and the bathroom wall, above the toilet). The room was then vacated, and the UV-C device was remotely activated. The room decontamination times were recorded for all cycles. Following cycle completion, Rodac plates containing DE neutralizing agar (Becton Dickinson) were used to culture each Formica template. These plates were then incubated aerobically at $37^{\circ} \mathrm{C}$ for 48 hours for MRSA and 
TAвLE 1. Ultraviolet C (UV-C) Decontamination (Mean $\log _{10}$ Reduction) of Formica Surfaces in a Patient Room That Were Experimentally Contaminated with Methicillin-Resistant Staphylococcus aureus (MRSA) and Clostridium difficile Spores with and without a Reflective Coating on Walls

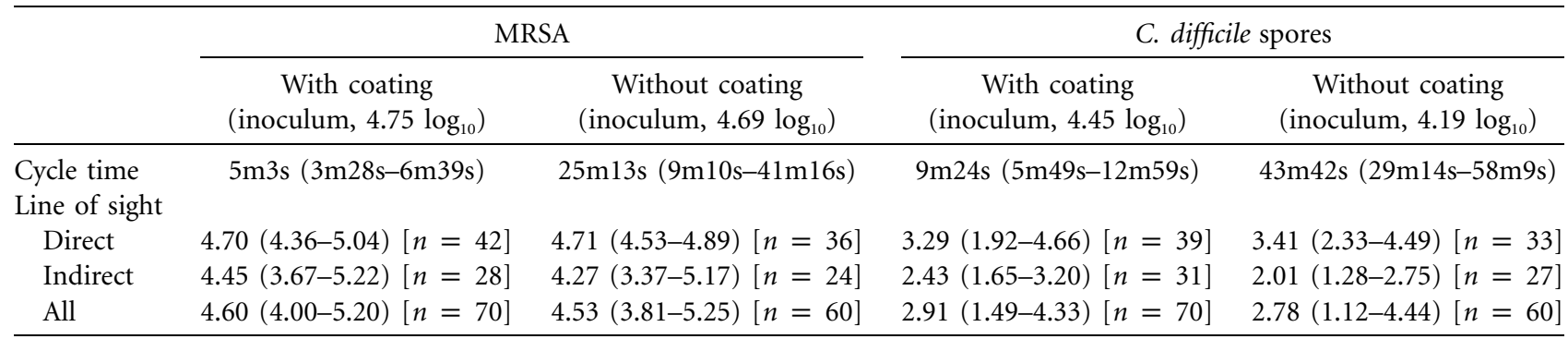

Note. The patient room comprised $130 \mathrm{ft}^{2}$. Data in parentheses are $95 \%$ confidence intervals, which were used to determine that there was no significant difference $(P>.05)$ when comparing cycle times with and without the reflective coating or when comparing direct UV-C to indirect UV-C. Sites were evaluated as receiving direct (laser point visible on site) or indirect (laser point not visible on site) UV-C by following the path of the laser. $n$ values indicate the number of samples.

anaerobically (Anaeropack; Mitsubishi Gas Chemical) at $37^{\circ} \mathrm{C}$ for 48 hours for $C$. difficile. After incubation, colony-forming units (CFUs) of the test organisms on each plate were quantified. The $C$. difficile culture was treated by heat at $56^{\circ} \mathrm{C}$ for 10 minutes, and the presence and resistance of $C$. difficile spores (not vegetative bacteria) was verified by exposing the stock preparation to dilute hydrochloric acid, as specified in the AOAC International sporicidal activity test. ${ }^{7}$ The suspension was then stained to confirm the presence of spores (more than $90 \%$ spores).

\section{RES ULTS}

In our unoccupied patient room, the effectiveness of UV-C radiation with respect to reduction of MRSA on surfaces was a $4.53-\log _{10}$ reduction without the reflective coating and a $4.60-\log _{10}$ reduction with the reflective coating $(P>.05)$, while the cycle time was 5 minutes 3 seconds with the reflective coating and 25 minutes 13 seconds without the reflective coating $(P<.05$; Table 1$)$. For C. difficile spores, there was a $2.78-\log _{10}$ reduction without the reflective coating and a $2.91-\log _{10}$ reduction with the reflective coating $(P>.05)$, but the cycle time with the reflective coating was 9 minutes 24 seconds, compared with 43 minutes 42 seconds without the reflective coating $(P<.05)$.

\section{DISCUSSION}

Several studies have shown the ability of the tested device to deliver lethal UV-C doses to epidemiologically important microorganisms, ${ }^{8-10}$ with pathogens reduced by $2.5 \log _{10}$ to more than $4 \log _{10}$ under high contamination levels that exceed those normally found in healthcare facilities. For example, studies have shown that, although the frequency of contamination by these pathogens (eg, C. difficile and MRSA) is high (10\% to more than $50 \%$ ), the microbial load is generally low (less than 10 CFUs per Rodac plate or sample), which suggests that the clinical efficacy of UV-C could be significant.
All room decontamination technologies have advantages and disadvantages. ${ }^{5}$ A major disadvantage of both UV and hydrogen peroxide systems is that they can be used only for terminal disinfection (ie, they cannot be used for daily disinfection) because the room must be emptied of people. The main advantage of both technologies is their ability to achieve a reduction in healthcare pathogens. A major disadvantage of hydrogen peroxide systems - and to a lesser extent UV$\mathrm{C}$ - is the time required for decontamination. The UV-C system offers faster decontamination (15-50 minutes, compared with 2-5 hours for hydrogen peroxide systems) before the development of this UV-C-reflective coating, but with this innovation cycle times were 5-10 minutes, which would significantly reduce (by approximately $80 \%$ ) the room's downtime before another patient could be admitted.

In summary, UV technology offers a potential option for room decontamination in healthcare settings. MRSA, VRE, multidrug-resistant Acinetobacter, and C. difficile spores comprise a growing reservoir of epidemiologically important pathogens that have an environmental mode of transmission. The UV-C technology (and other effective room decontamination technologies) can effectively reduce environmental contamination and potentially mitigate infection risks, and it should be considered when the environmental mode of transmission is important (eg, after patients under contact precautions are discharged) and enhanced interventions are not effective. Use of the nanostructured UV-C-reflective coating allows room decontamination to be completed in 5-10 minutes with UV-C, which could be easily integrated into healthcare practices in which the occupancy is high and fast patient room turnaround time is critical.

\section{ACKNOWLEDGMENTS}

We thank Lumalier for loaning the ultraviolet (UV) device used in these experiments to University of North Carolina (UNC) Health Care. We acknowledge the statistical assistance of Dr Emily E. Sickbert-Bennett and the 
use of rooms in the UNC Clinical Translational Research Center (National Institutes of Health 1ULRR025747-01).

Financial support. Lumalier loaned to UNC Health Care the UV device used in these experiments. No other financial or technical support was received by UNC Health Care.

Potential conflicts of interest. B.M.T. reports being a coinventor of the patent-pending reflective coating and a co-owner of Twilight Labs, which makes the reflective coating. All other authors report no conflicts of interest relevant to this article. All authors submitted the ICMJE Form for Disclosure of Potential Conflicts of Interest, and the conflicts that the editors consider relevant to this article are disclosed here.

Affiliations: 1. Hospital Epidemiology, University of North Carolina Health Care, Chapel Hill, North Carolina; 2. Division of Infectious Diseases, University of North Carolina School of Medicine, Chapel Hill, North Carolina; 3. Department of Chemical Engineering, University of North Dakota, Grand Forks, North Dakota.

Received September 26, 2012; accepted December 4, 2012; electronically published April 9, 2013.

(C) 2013 by The Society for Healthcare Epidemiology of America. All rights reserved. 0899-823X/2013/3405-0015\$15.00. DOI: 10.1086/670211

\section{REFERENCES}

$\rightarrow$ 1. Carling PC, Parry MF, Von Beheren SM; Healthcare Environmental Hygiene Study Group. Identifying opportunities to enhance environmental cleaning in 23 acute care hospitals. Infect Control Hosp Epidemiol 2008;29:1-7.

2. Huang SS, Datta R, Platt R. Risk of acquiring antibiotic-resistant bacteria from prior room occupants. Arch Intern Med 2006;166: 1945-1951.

3. Drees M, Snydman DR, Schmid CH, et al. Prior environmental contamination increases the risk of acquisition of vancomycinresistant enterococci. Clin Infect Dis 2008;46:678-685.

$\rightarrow$ 4. Shaughnessy MK, Micielli RL, DePestal DD, et al. Evaluation of hospital room assignment and acquisition of Clostridium difficile infection. Infect Control Hosp Epidemiol 2011;32:201-206.

$\rightarrow$ 5. Rutala WA, Weber DJ. Are room decontamination units needed to prevent transmission of environmental pathogens? Infect Control Hosp Epidemiol 2011;32:743-747.

6. Owens MU, Deal DR, Shoemaker MO, Knudson GB, Meszaros JE, Deal JL. High-dose ultraviolet C light inactivates spores of Bacillus subtilis var. niger and Bacillus anthracis Sterne on nonreflective surfaces. Appl Biosaf 2005;10:240-247.

7. Beloian A. Disinfectants. In: Cunniff P, ed. Official Methods of Analysis of the AOAC International. 16th ed. Gaithersburg, MD: AOAC International, 1999:6-1-6-18.

8. Rutala WA, Gergen MF, Weber DJ. Room decontamination with UV radiation. Infect Control Hosp Epidemiol 2010;31:1025-1029.

$\rightarrow$ 9. Boyce JM, Havill NL, Moore BA. Terminal decontamination of patient rooms using an automated mobile UV light unit. Infect Control Hosp Epidemiol 2011;32:737-742.

10. Nerandzic MM, Cadnum JL, Pultz MJ, Donskey CJ. Evaluation of an automated ultraviolet radiation device for decontamination of Clostridium difficile and other healthcare-associated pathogens in hospital rooms. BMC Infect Dis 2010;10:197. 